CARDIOVASCULAR MEDICINE

\title{
Assessment of infarcted myocardium with real time myocardial contrast echocardiography: comparison with technetium-99m sestamibi single photon emission computed tomography
}

\author{
P Tousek, M Orban, S Martinoff, C Firschke
}

See end of article for authors' affiliations

Correspondence to: Priv-Doz Dr med C Firschke, Deutsches Herzzentrum München, Lazarettstraße 36, D80636 Munich, Germany; cfirschke@t-online.de

Accepted

17 February 2005

Published Online First

17 March 2005

\begin{abstract}
Background: Little is known about the relation between the extent of microvascular damage and infarct size in patients after successful mechanical reperfusion of acute myocardial infarction.

Objective: To compare the spatial extent of reduced myocardial signal between real time myocardial contrast echocardiography (MCE) and single photon emission computed tomography (SPECT) after successful mechanical reperfusion of acute myocardial infarction and to test the hypothesis that MCE can be used for clinical infarct size assessment.

Methods: 10 days after successful mechanical reperfusion of acute myocardial infarction, 117 patients underwent MCE (power pulse inversion technique, slow contrast bolus injection) and SPECT (technetium$99 \mathrm{~m}$ sestamibi). Location and number of segments with normal myocardial signal intensity and with mild and severe reduction were registered and the concordance between the techniques was calculated.

Results: Segmental concordance between MCE and SPECT was $83 \%(\kappa=0.64)$. On average, the difference in the number of segments with reduced myocardial signal intensity between MCE and SPECT did not exceed one segment $(p<0.001)$. Sensitivity and specificity of MCE for the detection of an abnormal segment on SPECT were $87 \%$ and $91 \%$, respectively. Intraobserver and interobserver agreement were $94 \%(\kappa=0.84)$ and $92 \%(\kappa=0.83)$, respectively.

Conclusions: Real time MCE is a promising technique for infarct size assessment after successful mechanical reperfusion of acute myocardial infarction.
\end{abstract}

$\mathrm{T}$ he presence of myocardial microvascular damage despite successful reperfusion of acute myocardial infarction, myocardial "no reflow", can be imaged with myocardial contrast echocardiography (MCE) and has been shown to be an indicator of unfavourable clinical prognosis. ${ }^{12}$ Little is known, however, about the relation between the extent of myocardial microvascular and myocellular damage-that is, infarct size, one of the key characteristics of patients with myocardial infarction. ${ }^{3}{ }^{4}$ Whereas excellent agreement was obtained in the experimental setting, ${ }^{5}$ the results of previous clinical studies comparing intravenous MCE with single photon emission computed tomography (SPECT), the current reference standard of infarct size determination, were equivocal with some showing good and others poor agreement. ${ }^{6-10}$ Intravenous MCE, however, was in its infancy at the time of these studies and was highly susceptible to artefacts. The need for high ultrasound transmission power with a significant tissue background signal resulted in a narrow ratio of myocardial contrast to tissue signal, often preventing unequivocal image interpretation. Intermittent imaging, required to minimise ultrasound induced microbubble destruction, impeded the spatial orientation during echocardiography sometimes resulting in dubious comparability with SPECT. New contrast specific multipulse imaging algorithms such as power pulse inversion imaging, ${ }^{11-13}$ used in the present study, allow massive reduction of transmission power by up to $90 \%$ and almost exclusive imaging of the contrast signal. Owing to minimised microbubble destruction, perfusion studies can be performed in real time with simultaneous availability of regional wall motion information. We hypothesised that, based on these recent technical advances, good agreement would be obtained between the extent of myocardial microvascular damage on MCE and infarct size on SPECT after reperfusion of acute myocardial infarction.

\section{METHODS}

\section{Study population}

Patients with mechanical reperfusion of acute myocardial infarction who participated in the STOPAMI (stent versus thrombolysis for occluded coronary arteries in patients with acute myocardial infarction) I or II trial ${ }^{14}{ }^{15}$ were enrolled. The study protocol was approved by the institutional review board and all patients gave written informed consent. MCE was performed on the day of the SPECT study about 10 days after mechanical reperfusion in patients with adequate echocardiographic visualisation of the myocardium in the apical four and two chamber views.

\section{Myocardial contrast echocardiography}

Optison (Amersham Health, Oslo, Norway), a second generation ultrasound contrast agent consisting of perfluoropropane filled albumin microspheres (mean diameter $3.9 \mu \mathrm{m}$, concentration $5 \times 10^{8}-8 \times 10^{8} / \mathrm{ml}$ ), was used. For each echocardiographic view, $1 \mathrm{ml}$ of Optison was injected intravenously over 30 seconds through a multidirectional stopcock and $5 \mathrm{ml}$ of $0.9 \%$ saline were simultaneously injected to prevent microbubble floating.

An HDI-5500 ultrasound system (ATL Philips Medical Systems, Bothell, Washington, USA) equipped with a

Abbreviations: MCE, myocardial contrast echocardiography; SPECT, single photon emission computed tomography; STOPAMl, stent versus thrombolysis for occluded coronary arteries in patients with acute myocardial infarction 


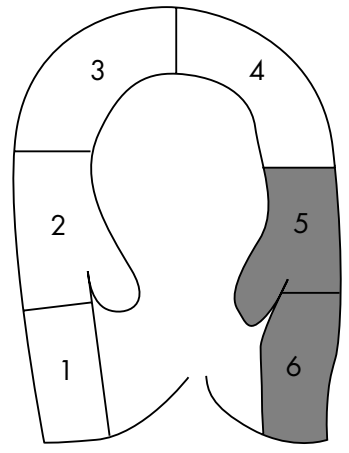

4 chamber view

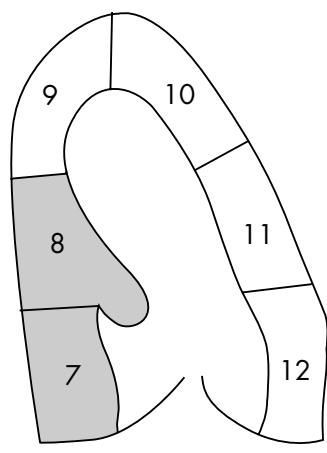

2 chamber view LAD

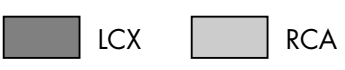

Figure 1 Myocardial segments and coronary perfusion territories of the left anterior descending coronary artery (LAD), left circumflex artery (LCX), and right coronary artery (RCA).

1.7 MHz transducer was used. Real time MCE was performed with power pulse inversion imaging. The dynamic range of this system was set to the high level and the frame rate during real time imaging was $15 \mathrm{~Hz}$. The gain settings were optimised at the beginning of each study and held constant throughout. The focus was set at the level of the mitral valve. Mechanical indices between 0.12 and 0.18 were used. Two and four chamber views were stored on S-VHS videotape.

\section{Single photon emission computed tomography}

SPECT was performed 45-60 minutes after intravenous injection of $500 \mathrm{MBq}$ of technetium-99m sestamibi. For image acquisition, a dual head rotating camera system (ADAC Vertex Plus, Milpitas, California, USA) with low energy, high resolution collimators was used. In a step and shoot acquisition mode, 32 images were acquired by clockwise eccentric rotation over a $180^{\circ}$ arc. Acquisition time of 40 seconds for each image and a $64 \times 64$ matrix were used. Images were reconstructed with a dedicated software package (Pegasys version 3.4, Pegasys Inc, Tokyo, Japan) and a Butterworth filter (order 5, 0.4 Nynquist cut off frequency). Tomographic slices reoriented to the short axis and to the vertical and horizontal long axes of the heart were generated. Vertical and horizontal long axis slices corresponding to the echocardiographic two and four chamber views were selected for comparison with MCE.

\section{Image interpretation}

A 12 segment model of the left ventricle was used for the analysis of wall motion, MCE, and SPECT (fig 1). Two observers blinded to both clinical and SPECT data interpreted regional wall motion and MCE. Similarly, two observers blinded to both clinical and echocardiographic data interpreted the SPECT data. Differences in opinion were resolved by consensus.

\section{Wall motion}

Regional wall motion was graded as 1, normal; 2, hypokinetic; or 3, akinetic.

\section{MCE}

Myocardial opacification in each of the 12 segments was graded as 1, normal; 2, mild reduction; or 3, severe reduction. Reduced myocardial opacification in segments with normal wall motion was assessed as artefact.

\section{SPECT}

The horizontal and vertical long axis planes closest to the echocardiographic apical two and four chamber views were used for interpretation. Tracer uptake was assessed in each of the 12 myocardial segments and graded as 1, normal; 2, mild reduction; or 3, severe reduction. Reduced myocardial tracer uptake in segments with normal wall motion was assessed as artefact. $^{16}$

Interobserver and intraobserver variability

Two observers separately scored 20 randomly selected sets of MCE data to determine interobserver variability. After two months, one of the observers repeated the reading and intraobserver variability was calculated.

\section{Statistical analysis}

Continuous variables are expressed as mean (SD); nominal variables are expressed as count and percentage. The agreement between MCE and SPECT scores and the interobserver and intraobserver agreement were characterised by $\kappa$ statistics. Values of $>0.4,>0.6$, and $>0.8$ indicate fair, good, and excellent agreement, respectively. ${ }^{17}$ Sensitivity and specificity of MCE for detection of regional signal reduction on SPECT were determined. The non-parametric signed ranked Kolmogorov-Smirnov test was used to test whether the difference in the number of segments with reduced myocardial signal between MCE and SPECT exceeded one segment. A probability value of $\mathrm{p}<0.05$ was considered significant.

\section{RESULTS}

\section{Patient characteristics}

We enrolled 117 patients in the study. For 14 patients, inadequate image quality on MCE prevented meaningful assessment of myocardial opacification. Three patients did not undergo SPECT. Table l lists the demographic, clinical, and angiographic characteristics of the 100 patients eligible for image analysis.

\section{Myocardial opacification and wall motion on echocardiography}

We used 1064 of 1200 segments (88\%) for final comparison. We excluded 66 segments $(6 \%)$ due to artefacts on MCE (myocardial opacification score 2 or 3 in the presence of normal contraction) and 58 segments $(5 \%)$ were excluded due to artefacts on SPECT (myocardial uptake score 2 or 3 in the presence of normal contraction). In 12 segments ( $1 \%$ ) artefacts were identified both on MCE and on SPECT and these segments were also excluded. On MCE, artefacts mainly occurred in basal segments of the anterior and lateral

\begin{tabular}{|c|c|}
\hline Age (years) & $63(12)$ \\
\hline Women & $22(22 \%)$ \\
\hline Weight (kg) & $79(13)$ \\
\hline Height $(\mathrm{cm})$ & $173(8)$ \\
\hline Q wave infarction & $73(73 \%)$ \\
\hline Multivessel disease & $73(73 \%)$ \\
\hline \multicolumn{2}{|l|}{ Infarct related artery } \\
\hline LAD & $50(50 \%)$ \\
\hline RCA & $33(33 \%)$ \\
\hline LCX & $17(17 \%)$ \\
\hline Arterial hypertension & $69(69 \%)$ \\
\hline Cholesterolaemia & $63(63 \%)$ \\
\hline Diabetes mellitus & $16(16 \%)$ \\
\hline History of smoking & $63(63 \%)$ \\
\hline Obesity & $38(38 \%)$ \\
\hline $\begin{array}{l}\text { Data are mean (SD) } \\
\text { LAD, left anterior des } \\
\text { circumflex coronary }\end{array}$ & $\begin{array}{l}\text { ary artery; LCX, left } \\
\text { ght coronary artery. }\end{array}$ \\
\hline
\end{tabular}



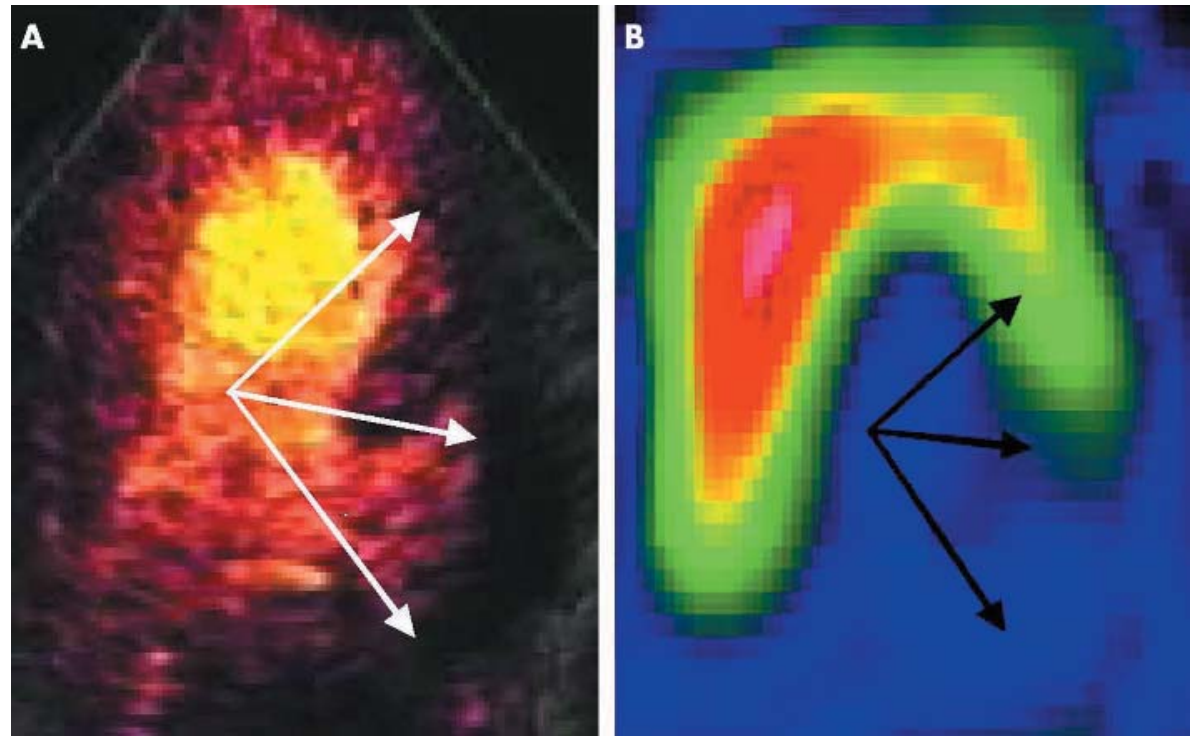

Figure 2 Reduction of myocardial signal intensity (arrows) in the lateral myocardium on myocardial contrast echocardiography (panel A) and single photon emission computed tomography (panel B). wall (34 of 66). On SPECT, artefacts mainly occurred in the inferior wall (34 of 58). Analysis of left anterior descending coronary artery and left circumflex artery perfusion territories was feasible for all patients. For four patients, the perfusion territory of the right coronary artery could not be analysed because of artefacts in both inferior segments.

Figures 2 depicts MCE and SPECT studies of a patient with previous lateral myocardial infarction.

MCE showed normal contractile function and normal myocardial opacification in 638 of 1122 (57\%) artefact-free segments on MCE. Myocardial opacification was also normal in $94(8 \%)$ hypokinetic and $13(1 \%)$ akinetic segments. In 377 (34\%) segments, myocardial opacification was reduced or absent in conjunction with contractile dysfunction.

\section{Myocardial signal magnitude on MCE and SPECT}

Table 2 shows agreement between MCE and SPECT with respect to myocardial signal classification as normal and mildly and severely reduced. The two techniques agreed in 881 of 1064 segments $(83 \%, \kappa=0.64)$. Concordance was highest $(91 \%, \kappa=0.68)$ in the mid septal segments and lowest in the basal lateral $(70 \%, \kappa=0.39)$ and mid inferior $(71 \%, \kappa=0.38)$ segments. By binary segmental classification as normal (score 1) or abnormal (score 2 or 3 ), concordance between MCE and SPECT reached 90\% $(\kappa=0.77)$.

Table 3 shows concordance between MCE and SPECT for normal (score 1) versus reduced myocardial signal (score 2 or 3 ) on the coronary perfusion territory level. Agreement was present in 251 of 296 territories $(85 \%, \kappa=0.71)$.

Table 2 Segmental semiquantitative signal comparison between myocardial contrast echocardiography (MCE) and single photon emission computed tomography (SPECT)

\begin{tabular}{llll}
\hline \multirow{2}{*}{$\begin{array}{l}\text { MCE (myocardial } \\
\text { opacification) }\end{array}$} & \multicolumn{2}{l}{ SPECT (myocardial sestamibi uptake) } \\
\cline { 2 - 4 } & Normal & Mild reduction & Severe reduction \\
\hline Normal & 675 & 30 & 11 \\
Mild reduction & 49 & 88 & 37 \\
Severe reduction & 15 & 41 & 118 \\
\hline
\end{tabular}

\section{Myocardial defect size on MCE and SPECT}

Figure 3 shows the relation between the number of segments with reduced myocardial signal on MCE and SPECT in each patient. On average, the difference in the number of segments with reduced myocardial signal between MCE and SPECT did not exceed one segment $(\mathrm{p}<0.001)$.

Sensitivity and specificity of MCE for the detection of an abnormal segment on SPECT were $87 \%$ and $91 \%$, respectively. Sensitivity and specificity of MCE on the perfusion territory level were $93 \%$ and $93 \%$ for the left anterior descending territory, $73 \%$ and $97 \%$ for the right coronary artery territory, and $97 \%$ and $81 \%$ for left circumflex artery territory, respectively.

\section{Observer agreement}

Intraobserver and interobserver agreement for differentiation of normal and mildly and severely reduced myocardial opacification on MCE were $94 \%(\kappa=0.84)$ and $92 \%$ $(\kappa=0.83)$, respectively.

\section{DISCUSSION}

The present study for the first time shows that the extent of microvascular damage after mechanical reperfusion of acute myocardial infarction, shown on real time MCE, is closely correlated with infarct size. Real time MCE may therefore allow bedside assessment of infarct extent after mechanical reperfusion of acute myocardial infarction.

\section{Physiology of myocardial perfusion imaged with MCE and SPECT}

The myocardial contrast signal on MCE and sestamibi signal on SPECT reflect overlapping but not identical physiological aspects of myocardial perfusion. Myocardial opacification on MCE indicates the presence of microbubbles, pure intravascular tracers, in the myocardial microvasculature. This signal is therefore exclusively related to myocardial blood flow. ${ }^{99 \mathrm{~m}} \mathrm{Tc}$-sestamibi, in contrast, diffuses into the extravascular space, passively enters the myocyte, and binds to the negatively charged mitochondrial membrane. The myocardial sestamibi signal on SPECT, therefore, requires not only intact blood flow to the myocardium but also myocardial cell integrity-that is, preserved mitochondrial membrane potential. Thus, the correlation between MCE and SPECT in the present study shows a physiological link between 
Table 3 Signal comparison between MCE and SPECT by myocardial perfusion territories

\begin{tabular}{|c|c|c|c|c|c|c|}
\hline \multirow{3}{*}{$\begin{array}{l}\text { MCE (myocardial } \\
\text { opacification) }\end{array}$} & \multicolumn{6}{|c|}{ SPECT (myocardial sestamibi uptake) } \\
\hline & \multicolumn{2}{|l|}{ LAD } & \multicolumn{2}{|l|}{ RCA } & \multicolumn{2}{|l|}{ LCX } \\
\hline & Normal & Abnormal & Normal & Abnormal & Normal & Abnorma \\
\hline Normal & 23 & 7 & 32 & 13 & 52 & 1 \\
\hline Abnormal & 8 & 62 & 1 & 50 & & 32 \\
\hline Concordance ( $\mathrm{k}$ ) & \multicolumn{2}{|c|}{$85 \%(0.65)$} & \multicolumn{2}{|c|}{$85 \%(0.63)$} & \multicolumn{2}{|c|}{$84 \%(0.67)$} \\
\hline
\end{tabular}

microvascular and myocellular damage after mechanical reperfusion of acute myocardial infarction.

The time interval of 10 days after mechanical reperfusion may have contributed to the good correlation between MCE and SPECT, since dynamic signal changes early after reperfusion probably have abated by that time. ${ }^{18}$

\section{Technical aspects of MCE}

In previous clinical studies comparing intravenous MCE with radionuclide imaging, second harmonic ${ }^{679}{ }^{19}$ and power Doppler imaging ${ }^{8}{ }^{10}$ were used. In the multicentre studies of Marwick et $a l^{7}$ and Binder et $a l^{6}$ sensitivities of MCE for the detection of abnormal segments on SPECT at rest were low and ranged from $14-65 \%$ and from $20-39 \%$, respectively, depending on the dose of the contrast agent. Jucquois et al ${ }^{9}$ obtained the best agreement between SPECT and MCE. In their study, the overall agreement between the techniques was $82 \%(\kappa=0.65)$. Sensitivity and specificity of MCE for the detection of abnormal segments on SPECT were $76 \%$ and $83 \%$, respectively. Their study, however, comprised a relatively small number of patients and $26 \%$ of all segments could not be used for comparison with SPECT due to artefacts on MCE.

We attribute the improved concordance between MCE and SPECT of the present study to the increased signal to noise ratio of the power pulse inversion technique. ${ }^{13}$ This assumption is supported by the comparably low rate of uninterpretable segments in the present study. Furthermore, MCE is simplified by the real time feasibility of power pulse inversion technique improving spatial orientation during the examination, which, in its own right, may have contributed to the excellent concordance with SPECT in the present study. After all, the recognition of MCE artefacts is facilitated by the simultaneous assessment of regional myocardial perfusion

SPECT

(number of abnormal segments in a patient)

\begin{tabular}{|c|c|c|c|c|c|c|c|c|c|c|c|}
\hline$\overline{\bar{\tau}}$ & & 0 & 1 & 2 & 3 & 4 & 5 & 8 & 6 & 7 & 9 \\
\hline & 0 & 1 & 4 & 2 & - & - & - & - & - & - & - \\
\hline & 1 & 2 & 3 & 3 & 1 & - & _ & - & - & - & - \\
\hline & 2 & 1 & 4 & 3 & 2 & 1 & _ & 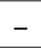 & - & - & - \\
\hline & 3 & - & - & 4 & 4 & 5 & 1 & . & - & - & - \\
\hline & 4 & - & - & 1 & 4 & 4 & 3 & 1 & - & 1 & 1 \\
\hline & 5 & - & 1 & - & 3 & 2 & 4 & 4 & 2 & - & 1 \\
\hline & 6 & - & - & - & 1 & 3 & 5 & 3 & 5 & 1 & - \\
\hline & 7 & - & - & - & - & - & 1 & 1 & 3 & 2 & - \\
\hline & 8 & - & - & - & - & - & - & - & - & - & - \\
\hline & 9 & - & - & - & - & - & - & - & - & - & 1 \\
\hline
\end{tabular}

Figure 3 Number of patients with concordant and discordant spatial extent of reduced myocardial signal intensity. and wall motion. Disadvantages of power pulse inversion technique, on the other hand, include a limited dynamic range, which may have prevented closer correlation between MCE and SPECT in the present study.

\section{Study limitations}

The imaging data were analysed only qualitatively in the present study and more accuracy might have been achieved by signal quantification based on signal intensity thresholds.

In contrast with continuous infusion of the contrast agent, bolus administration as used in the present study increases the risk of attenuation artefacts on MCE.

Echocardiographic two or four chamber views may not accurately match horizontal and vertical long axis SPECT planes selected for comparison, which may have resulted in erroneous segmental association between the two techniques.

The applicability of the present data is limited to patients with adequate visualisation of all myocardial segments on the baseline echocardiogram.

\section{Conclusions and clinical prospects}

Infarct size is not widely determined after reperfusion of acute myocardial infarction because SPECT imaging is not readily available in most hospitals. The close physiological link between myocardial microvascular and myocellular damage after mechanical reperfusion of acute myocardial infarction, shown in the present study, may help to establish the use of real time MCE for infarct size assessment. Real time MCE has the advantage of being a practicable bedside technique with the potential of wide availability. On the basis of the present data, it appears reasonable to evaluate the role of real time MCE for risk stratification after acute myocardial infarction in future studies. In light of increasing numbers of reperfusion strategies for patients with acute coronary syndromes and existing highly effective treatment modalities, real time MCE may serve as versatile tool for rational and cost effective comparison of efficacy differences between the modalities and may guide the planning of large scale mortality trials.

\section{Authors' affiliations \\ P Tousek, M Orban, S Martinoff, C Firschke, Deutsches Herzzentrum München, Munich, Germany}

Dr Tousek was supported by a grant from the German Cardiac Society, Düsseldorf, Germany. Dr Orban was supported by a grant from the Czech Cardiac Society, Brno, Czech Republic.

\section{REFERENCES}

1 Ito $\mathrm{H}$, Tomooka T, Sakai N, et al. Lack of myocardial perfusion immediately after successful thrombolysis: a predictor of poor recovery of left ventricular function in anterior myocardial infarction. Circulation 1992;85:1699-705.

2 Andrassy P, Zielinska M, Busch R, et al. Myocardial blood volume and the amount of viable myocardium early after mechanical reperfusion of acute myocardial infarction: prospective study using venous contrast echocardiography. Heart 2002;87:350-5. 
3 Miller TD, Christian TF, Hopfenspirger MR, et al. Infarct size after acute myocardial infarction measured by quantitative tomographic $99 \mathrm{mTc}$ sestamibi imaging predicts subsequent mortality. Circulation 1995;92:334-41.

4 Miller TD, Hodge DO, Sutton JM, et al. Usefulness of technetium- $99 \mathrm{~m}$ sestamibi infarct size in predicting posthospital mortality following acute myocardial infarction. Am J Cardiol 1998:81:1491-3.

5 Firschke C, Lindner JR, Wei K, et al. Myocardial perfusion imaging in the setting of coronary artery stenosis and acute myocardial infarction using venous injection of a second-generation echocardiographic contrast agent. Circulation 1997:96:959-67.

6 Binder T, Assayag P, Baer F, et al. NC100100, a new echo contrast agent for the assessment of myocardial perfusion-safety and comparison with technetium-99m sestamibi single-photon emission computed tomography in a randomized multicenter study. Clin Cardiol 1999;22:273-82.

7 Marwick TH, Brunken R, Meland N, et al. Accuracy and feasibility of contrast echocardiography for detection of perfusion defects in routine practice: comparison with wall motion and technetium-99m sestamibi single-photon emission computed tomography. J Am Coll Cardiol 1998;32:1260-9.

8 Haluska B, Case C, Short L, et al. Effect of power Doppler and digital subtraction techniques on the comparison of myocardial contrast echocardiography with SPECT. Heart 2001;85:549-55.

9 Jucquois I, Nihoyannopoulos P, D'Hondt A-M, et al. Comparison of myocardial contrast echocardiography with $\mathrm{NC100100}$ and ${ }^{99 m} \mathrm{Tc}$ sestamibi SPECT for detection of resting myocardial perfusion abnormalities in patients with previous myocardial infarction. Heart 2000;83:518-24.

10 Heinle SH, Noblin J, Goree-Best P, et al. Assessment of myocardial perfusion by harmonic power Doppler imaging at rest and during adenosine stress: comparison with $99 \mathrm{mTc}$-sestamibi SPECT imaging. Circulation $2000 ; 102: 55-60$.
11 Tiemann K Lohmeier S, Kuntz S, et al. Real-time contrast echo assessment of myocardial perfusion at low emission power: first experimental and clinical results using power pulse inversion imaging. Echocardiography 1999; 16:799-809.

12 Mor-Avi V, Caiani EG, Collins KA, et al. Combined assessment of myocardial perfusion and regional left ventricular function by analysis of contrast-enhanced power modulation images. Circulation 2001; 104:352-7

13 Simpson DH, Burns PN. Pulse inversion Doppler: a new method for detecting nonlinear echoes from microbubble contrast agents. IEEE Trans Ultrason Ferroelect Freq Contr 1999;46:372-82.

14 Schömig A, Kastrati A, Dirschinger J, et al. Coronary stenting plus platelet glycoprotein Ilb/llla blockade compared with tissue plasminogen activator in acute myocardial infarction. N Engl J Med 2000;343:385-91.

15 Kastrati A, Mehilli J, Dirschinger J, et al. Myocardial salvage after coronary stenting plus abciximab versus fibrinolysis plus abciximab in patients with acute myocardial infarction. Lancet 2002;359:920-5.

16 DePuey EG, Garcia EV. Optimal specificity of thallium-201 SPECT through recognition of imaging artifacts. J Nucl Med 1989:30:441-9.

17 Kramer MS, Feinstein AR. Clinical biostatistics. LIV. The biostatistics of concordance. Clin Pharmacol Ther 1981;29:111-23.

18 Galiuto L, Lombardo A, Maseri A, et al. Temporal evolution and functional outcome of no reflow: sustained and spontaneously reversible patterns following successful coronary recanalisation. Heart 2003;89:731-7.

19 Shimoni S, Zoghbi WA, Xie F, et al. Real-time assessment of myocardial perfusion and wall motion during bicycle and treadmill exercise echocardiography: comparison with single photon emission computed tomography. J Am Coll Cardiol 2001;37:741-7.

\section{IMAGES IN CARDIOLOGY}

\section{Osler-Weber-Rendu syndrome}

A 54 year old white woman was admitted with a 10 year history of progressive dyspnoea and exertional hypoxia (oxygen saturation fell from $96 \%$ to $77 \%$ after two minutes of exercise). The typical clinical stigmata of periungual erythema/telangiectasias and telangiectasias of both the conjunctivae reflection (panel A) and inner mucosa of the lip were noted. A presumptive diagnosis of hereditary haemorrhagic telangiectasia (OslerWeber-Rendu syndrome) was made and pulmonary artery catheterisation confirmed a right to left shunt. Pulmonary angiography revealed multiple arterial venous malformations (AVMs) (panel B). On further questioning, the patient described a history of worsening headaches that had been diagnosed as "atypical migraines". Cerebral angiography confirmed a $1 \times 1 \times 1 \mathrm{~cm}$ compact nidus left occipital lobe AVM (panel C). The patient clinically improved after pulmonary coil
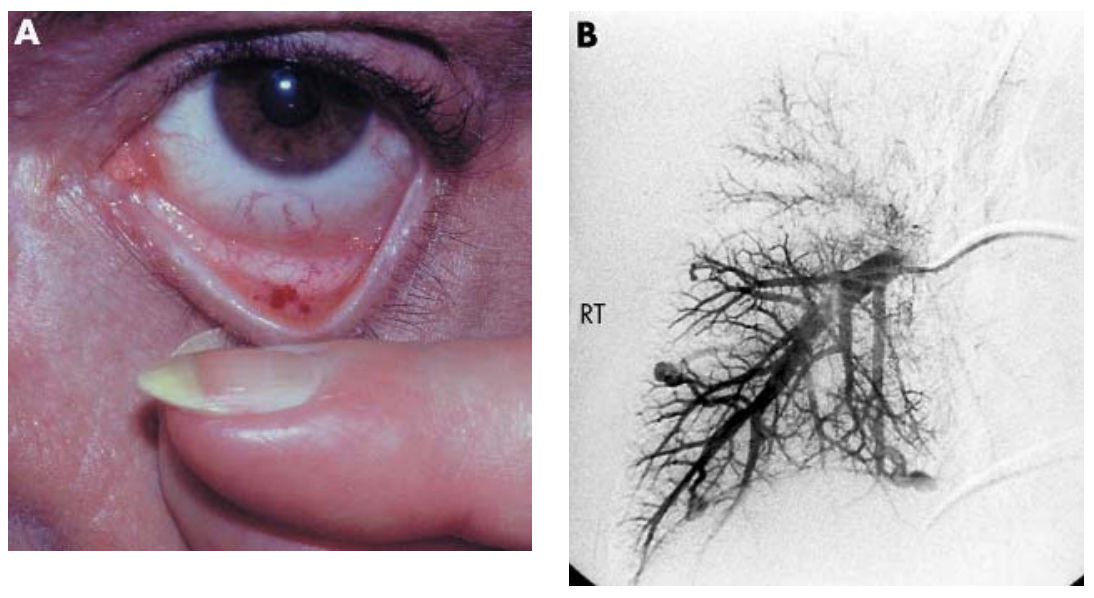

embolisation and gamma knife ablation of the cerebral AVM.

M C Bates

A Almehmi mark.bates@camc.org

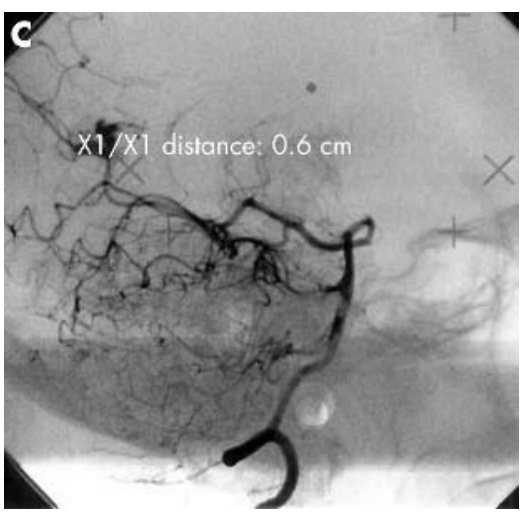

